\title{
Differential Effects of Laparoscopic Sleeve Gastrectomy and Laparoscopic Gastric Bypass on Appetite, Circulating Acyl-ghrelin, Peptide YY3-36 and Active GLP-1 Levels in Non-diabetic Humans
}

\author{
Ahmed Yousseif • Julian Emmanuel • Efthimia Karra • Queensta Millet • \\ Mohamed Elkalaawy • Andrew D. Jenkinson • Majid Hashemi • Marco Adamo • \\ Nicholas Finer • Alberic G. Fiennes • Dominic J. Withers • Rachel L. Batterham
}

Published online: 31 August 2013

(C) The Author(s) 2013. This article is published with open access at Springerlink.com

\begin{abstract}
Laparoscopic Roux-en-Y gastric bypass (LRYGBP) reduces appetite and induces significant and sustainable weight loss. Circulating gut hormones changes engendered by LRYGBP are implicated in mediating these beneficial effects. Laparoscopic sleeve gastrectomy (LSG) is advocated as an alternative to LRYGBP, with comparable short-term weight loss and metabolic outcomes. LRYGBP and LSG are anatomically
\end{abstract}

Ahmed Yousseif and Julian Emmanuel contributed equally.

A. Yousseif · J. Emmanuel • E. Karra • Q. Millet •

R. L. Batterham $(\bowtie)$

Centre for Obesity Research Department of Medicine, University

College London, Rayne Institute, 5, University Street,

WC1E 6JJ London, UK

e-mail: r.batterham@ucl.ac.uk

A. Yousseif $\cdot$ M. Elkalaawy $\cdot$ A. D. Jenkinson $\cdot$ M. Hashemi

M. Adamo $\cdot$ N. Finer $\cdot$ R. L. Batterham

Centre for Weight Loss, Metabolic and Endocrine Surgery,

University College London Hospitals, 250 Euston Road,

NW1 2BU London, UK

\section{Elkalaawy}

Clinical and Experimental Surgery Department, Medical Research Institute, University of Alexandria, Hadara, Alexandria, Egypt

\section{A. G. Fiennes}

Surrey Weight Loss Centre, St Anthony's Hospital North, Cheam SM3 9DW, UK

\section{J. Withers}

Metabolic Signalling Group, Medical Research Council Clinical Sciences Centre, Imperial College, W12 0NN London, UK

\section{R. L. Batterham}

Biomedical Research Centre, National Institute of Health Research University College London Hospitals, W1T 7DN London, UK distinct procedures causing differential entero-endocrine cell nutrient exposure and thus potentially different gut hormone changes. Studies reporting the comparative effects of LRYGBP and LSG on appetite and circulating gut hormones are controversial, with no data to date on the effects of LSG on circulating peptide YY3-36 (PYY3-36) levels, the specific PYY anorectic isoform. In this study, we prospectively investigated appetite and gut hormone changes in response to LRYGBP and LSG in adiposity-matched non-diabetic patients. Anthropometric indices, leptin, fasted and nutrient-stimulated acyl-ghrelin, active glucagon-like peptide-1 (GLP-1), PYY3-36 levels and appetite were determined pre-operatively and at 6 and 12 weeks postoperatively in obese, non-diabetic females, with ten undergoing LRYGBP and eight adiposity-matched females undergoing LSG. LRYGBP and LSG comparably reduced adiposity. LSG decreased fasting and post-prandial plasma acyl-ghrelin compared to pre-surgery and to LRYGBP. Nutrient-stimulated PYY3-36 and active GLP-1 concentrations increased postoperatively in both groups. However, LRYGBP induced greater, more sustained PYY3-36 and active GLP-1 increments compared to LSG. LRYGBP suppressed fasting hunger compared to LSG. A similar increase in post-prandial fullness was observed post-surgery following both procedures. LRYGBP and LSG produced comparable enhanced satiety and weight loss. However, LSG and LRYGBP differentially altered gut hormone profiles.

Keywords Laparoscopic Roux-en-Y gastric bypass (LRYGBP) · Laparoscopic sleeve gastrectomy (LSG) · Appetite · Acyl-ghrelin · Peptide YY3-36 (PYY3-36) · Active glucagon-like petide-1 (GLP-1) 


\section{Introduction}

Obesity is a leading cause of morbidity and mortality, placing growing demands on healthcare systems. Bariatric surgery induces significant long-lasting weight loss, ameliorates obesity-associated co-morbidities and reduces mortality [1]. Laparoscopic Roux-en-Y gastric bypass (LRYGBP) is the 'gold standard' procedure, resulting in 65-80\% excess bodyweight loss, decreased appetite, and rapid weightindependent amelioration of type-2 diabetes mellitus (T2DM) [2-5]. LRYGBP is cost-effective, but technically challenging with associated mortality — albeit low 0.09\% [6] — and micronutrient deficiencies risks, necessitating lifelong followup. Laparoscopic sleeve gastrectomy (LSG) (originally undertaken as a first-step in super-obese patients with subsequent conversion to a hybrid restrictive-malabsorptive procedure) is technically less complex, with lower complications and nutritional deficiencies rates than LRYGBP [2]. In light of reports of comparable weight loss and metabolic outcomes to LRYGBP, LSG is increasingly undertaken as a stand-alone procedure [7-10]. However, its long-term efficacy for weight loss and metabolic benefit remains unclear $[8,10]$.

Understanding the mechanisms mediating the weight loss and metabolic effects of bariatric surgery is key for developing less invasive procedures and medical obesity treatments. Postoperative changes in circulating gut hormones, including ghrelin, peptide YY (PYY) and glucagon-like peptide-1 (GLP-1), are thought to play a key role to the beneficial outcomes of bariatric surgery [2].

Ghrelin is produced by $X / A$-like cells predominantly located in the stomach fundus and proximal small intestine. Its circulating levels increase with fasting and decrease postprandially. Acyl-ghrelin, the bioactive form, exerts orexigenic properties and is produced by ghrelin octanoylation in serine3 mediated by ghrelin-O-acyl transferase (GOAT). Acylghrelin is rapidly converted by endogenous esterases to the main circulating form, des-acyl-ghrelin [11]. Moreover, ghrelin is labile, with highest stability in acidic states [12]. Thus, plasma acyl-ghrelin assessment requires specific sample processing by addition of esterase inhibitor and plasma acidification [13].

GLP-1 and PYY are released post-prandially by distal gut entero-endocrine L-cells [2]. Bioactive forms of GLP-1 augment glucose-dependent insulin release and decrease appetite [2]. Active GLP-1 is rapidly inactivated by the protease dipeptidyl-peptidase-4 (DPP-4). PYY3-36 reduces appetite, bodyweight and adiposity and is produced by truncation of the full-length PYY1-36 by DPP-4 [14]. The effects of PYY136 on appetite and feeding are less clear. In rodents, central PYY1-36 administration stimulates feeding [15], whereas equipotent or weaker anorexigenic effects to PYY3-36 have been attributed to peripheral PYY1-36 administration [16, 17]. However, to date, there is no evidence that systemically administered PYY1-36 alters human appetite [18]. Accurate assessment of plasma active GLP-1 and PYY3-36 requires rapid addition of DPP4-inhibitor to samples [13]. Dietinduced weight loss increases ghrelin and reduces PYY, but has little effect on GLP-1 [4, 19, 20], whilst in post-LRYGBP despite the weight loss, ghrelin decreases and marked, weightindependent, increments in nutrient-stimulated plasma PYY and GLP-1 occur [2-5, 21-24].

LRYGBP and LSG differentially alter gastrointestinal anatomy. LRYGBP reduces stomach volume and bypasses the majority of the stomach, duodenum and proximal jejunum, with direct nutrient delivery to the distal gut [2]. In LSG, the gastric fundus (the major source of ghrelin source) is excised, and this accelerates gastric emptying and intestinal transit post-operatively resulting in rapid nutrient delivery to the duodenum and hindgut [25-27]. Thus, LSG and LRYGBP produce differential nutrient exposure of entero-endocrine cells, and as such would be anticipated to differentially alter circulating gut hormones. Most studies comparing the two procedures report larger decreases in circulating fasting and/or meal-stimulated ghrelin after LSG versus LRYGBP [21, 28-30]; however, findings of their effects on hindgut hormones have been inconsistent, reporting either comparable increases in GLP-1 and/or total-PYY [8, 21, 28, 29, 31]; or superior total-PYY [32] and GLP-1 [30] increases postLRYGBP versus LSG. Technical procedural variations, differences in hormonal isoforms assessed, time-lapse from surgery, subjects' HOMA-IR and glycaemic status, and differences in subject standardization and sample processing may account for these discrepancies.

Several studies have measured total-ghrelin, total-GLP-1 and/or total-PYY post-surgery, which depict hormone production, but may not necessarily reflect circulating levels of their respective bioactive forms. In support of this, DPP-4 activity declines after LRYGBP [33], whereas GOAT expression is altered by caloric restriction and plasma GOAT is BMIdependent and thus may change post-bariatric surgery [34-36]. Moreover, the effects of LSG on the anorectic PYY-isoform, PYY3-36, are unknown. Therefore, we prospectively compared the effects of LSG and LRYGBP on anthropometric indices, leptin, acyl-ghrelin, active GLP-1, PYY3-36, and appetite in non-diabetic patients using our established subject standardisation and stringent sampleprocessing protocols.

\section{Materials and Methods}

\section{Subjects}

Ten obese non-diabetic females undergoing LRYGBP (age= $46.8 \pm 1.5$ years, body mass index $[\mathrm{BMI}]=45 \pm 1.5 \mathrm{~kg} / \mathrm{m}^{2}$ ) and eight adiposity-matched non-diabetic females undergoing 
LSG $\left(\right.$ age $=41.4 \pm 3.4$ years, BMI $=44 \pm 1.6 \mathrm{~kg} / \mathrm{m}^{2}$ ) were recruited. Inclusion criteria were female sex (for purpose of sex matching), BMI $=40-50 \mathrm{~kg} / \mathrm{m}^{2}$, aged $18-60$ years, and undergoing their first bariatric procedure. Exclusion criteria were T2DM, smoking, alcohol consumption $>20$ units/week and intra-operative/ early post-operative complications. Subjects gave written consent, and approval was obtained from University College London Hospitals Ethics Committee (project 09/H0715/65).

\section{Surgical Procedures}

LRYGBP included an antecolic-antegastric Roux-en-Y construction with $120-\mathrm{cm}$ alimentary/80-cm biliopancreatic limbs and a small vertical gastric pouch. LSG was performed as previously described [37] and calibrated tightly with a 32-Ch bougie with stapling commenced $5 \mathrm{~cm}$ from the pylorus.

\section{Study Protocol}

Patients attended for a 500-kcal test meal (43\% carbohydrate/ $18 \%$ protein $/ 39 \%$ fat) within 2 weeks pre-surgery and at 6 and 12 weeks ( $6 \mathrm{w}$ and $12 \mathrm{w}$ ) post-operatively. Subjects maintained similar food intake, refrained from alcohol $24 \mathrm{~h}$ prior to each study day, fasted from 9 p.m. and drank only water. At $\sim 9$ a.m. on study days, a peripheral cannula was inserted, and $1 \mathrm{~h}$ was allowed for recovery/habituation [13]. At t0 min, a baseline visual analogue scale (VAS) [13] and a blood sample were collected. Subjects consumed the test meal ( $250 \mathrm{ml}$ Resource2.0+fibre, Nestle Nutrition, Croydon, UK) within $15 \mathrm{~min}$, with blood drawn at t15, t30 and every $30 \mathrm{~min}$ thereafter until t180 min post-meal. Coincident with blood sampling, subjects completed appetite VAS [13]. Samples were processed by previously described stringent protocols [13].

\section{Anthropometric Phenotyping}

Height was determined by a wall-mounted stadiometer (242 Measuring Rod, Seca, UK), and body composition by multifrequency bioimpedance (InBody_720, Biospace, Derwent Healthcare, UK). Excess weight (EW) was calculated by subtracting ideal bodyweight (based on BMI $=25 \mathrm{~kg} / \mathrm{m}^{2}$ ) from pre-operative bodyweight. Percent excess weight loss $(\% \mathrm{EWL})$ was calculated as: $[100 \times($ bodyweight pre-surgery - current bodyweight)/excess weight].

\section{Hormone Assays}

To limit inter-assays' variability, samples were processed simultaneously. Plasma insulin, PYY3-36 and acyl-ghrelin were assayed by radioimmunoassay (assays' characteristics, respectively: sensitivity $2 \mu \mathrm{U} / \mathrm{mL}, 20 \mathrm{pg} / \mathrm{mL}, 7.8 \mathrm{pg} / \mathrm{ml}$; inter-assay variability, 3.8, 6.6, and $6.8 \%$; intra-assay variability, $2.6,4.5$, and $5 \%$ ). Plasma leptin and active GLP-1 were assayed by ELISA (respectively: sensitivity $0.5 \mathrm{ng} / \mathrm{mL}, 2 \mathrm{pM}$; inter-assay variability N/A, $7.5 \%$; intra-assay variability, 3.2 and $5.2 \%$ ) (radioimmunoassays and ELISAs by Millipore, Watford, UK). Plasma glucose was determined by enzymatic colorimetric assay, Infinity ${ }^{\mathrm{TM}}$ Glucose Oxidase Reagent (sensitivity $3.3 \mathrm{mmol} / \mathrm{L}$; ThermoFisher, Loughborough, UK). Insulin resistance was calculated using the homeostasis model assessment for insulin resistance, HOMA-IR [38].

\section{Statistical Analysis}

Results are expressed as mean \pm standard error of the mean. Normal distribution was assessed by D'Agostino-Pearson omnibus normality test. Integrated area-under-the-curve (AUC) $\mathrm{t} 0-\mathrm{t} 180$ for appetite and hormone concentrations versus time was calculated using the trapezoid rule. Non-paired student's $t$ test was used for between-groups comparisons, and paired student's $t$ test and repeated measures one-way ANOVA with Bonferroni post-hoc for within-groups' analysis. $p<0.05$ was considered significant.

\section{Results}

\section{Preoperative Patient Characteristics}

The two groups had comparable age, BMI and adiposity and exhibited similar circulating fasting leptin, acyl-ghrelin, PYY336, active GLP-1, glucose, insulin levels and HOMA-IR at baseline (Fig. 1, Tables 1 and 2). In response to the test meal, we observed decreases in hunger and acyl-ghrelin and increases in perceived satiety and in plasma PYY3-36, active GLP-1, glucose, and insulin, with no between-groups differences (Fig. 1, Tables 1 and 2).

Post-surgery Changes in Adiposity and Plasma Leptin

Both procedures induced comparable, marked reductions in BMI, fat mass, visceral fat area (VFA) and fasting leptin, with similar BMI and \%EWL observed at $6 \mathrm{w}$ and $12 \mathrm{w}$ postsurgery (Tables 1 and 2).

Post-operative Fasting and Nutrient-Stimulated Plasma Acyl-ghrelin Levels

Post-LRYGBP fasting acyl-ghrelin non-significantly decreased at $6 \mathrm{w}(p=0.06)$, but rose towards pre-surgery values by $12 \mathrm{w}(p=0.74)$. Post-meal, plasma acyl-ghrelin levels were reduced compared to pre-surgery at $\mathrm{t} 30$ at $6 \mathrm{w}$ and $12 \mathrm{w}$ postoperatively, whereas acyl-ghrelinAUC ${ }_{0-180}$ remained unaltered (Fig. 2, Table 2). At both post-LSG visits, lower fasting acyl- 
ghrelin levels and nutrient-stimulated levels at $\mathrm{t} 30$ and $\mathrm{t} 60$ were observed, with a trend towards decreased acyl-ghrelinAUC $\mathrm{C}_{0-}$ ${ }_{180}(p<0.1)$ compared to pre-surgery (Table 2$)$. Fasting acylghrelin and acyl-ghrelinAUC $\mathrm{A}_{0-180}$ were significantly lower post-LSG versus post-LRYGBP (Fig. 2, Table 2).

Post-operative Fasting and Nutrient-Stimulated Plasma PYY3-36 and Active GLP-1 Levels

Fasting PYY3-36 and active GLP-1 did not significantly change following either procedure (Fig. 3, Table 2). However, marked augmentation of nutrient-stimulated PYY3-36 and active GLP-1 occurred post-operatively (Fig. 3) (LRYGBP: PYY3-36 6w and 12w: t15-t180, active GLP-1 6w: t15t180, 12w: t15-t150; LSG: PYY3-36 6w: t15-t180, 12w: t15-t150, active GLP-1 6w: t15-t90, 12w: t30-t120), with associated increases in PYY3-36AUC $0-180$ and active GLP$1 \mathrm{AUC}_{0-180}$ (Fig. 3, Table 2) (LRYGBP: PYY3-36AUC $0-180$ $6 \mathrm{w}=\uparrow 2.5 \pm 0.2$-fold, $12 \mathrm{w}=\uparrow 2.3 \pm 0.3$-fold, active GLP- $1 \mathrm{AUC}_{0-}$ 180: $6 \mathrm{w} \& 12 \mathrm{w}=\uparrow 5.1 \pm 0.8$-fold; LSG: PYY3-36AUC ${ }_{0-180} 6 \mathrm{w}$ and $12 \mathrm{w}=\uparrow 1.7 \pm 0.2$-fold, active GLP- $1 \mathrm{AUC}_{0-180:} 6 \mathrm{w}=\uparrow 3.1 \pm$ 0.4 -fold, $12 \mathrm{w}=\uparrow 2.4 \pm 0.3$-fold). The LRYGBP group exhibited significantly higher PYY3-36AUC ${ }_{0-180}$ and active GLP$1 \mathrm{AUC}_{0-180}$, with higher meal-stimulated levels versus LSG (PYY3-36, 6w: t90-t150, 12w: t120-150; active GLP-1 6w: t15-t30, 12w: t30-t180) (Fig. 3, Table 2).

\section{Post-operative Subjective Hunger and Satiety Scores}

Fasting hunger significantly decreased post-LRYGBP, but remained unchanged post-LSG. Both procedures comparably reduced hungerAUC $\mathrm{A}_{0-180}$ in response to the standard test meal (Fig. 4, Table 2). At 12w, fasting hunger was lower postLRYGBP versus LSG, with no other between-groups differences in hunger (Fig. 4, Table 2).

Fasting fullness non-significantly declined at $6 \mathrm{w}(p=0.06)$, but returned to baseline by $12 \mathrm{w}$ post-LRYGBP, whereas remained unchanged post-LSG. Both procedures comparably enhanced postprandial fullness (Fig. 4, Table 2).

\section{Discussion}

LRYGBP and LSG reduced BMI, excess weight, adiposity and plasma leptin at $6 \mathrm{w}$ and $12 \mathrm{w}$ post-operatively to a similar extent. Hence, the observed differences in appetite and gut hormones were not attributable to differences in weight loss and more likely reflect differences in the surgical procedures per se.

Our study reports the first comparison of plasma acylghrelin in non-diabetic patients' post-LRYGBP and LSG. These procedures produce differential nutrient contact with ghrelin-producing $X / A$-like cells. Post-LRYGBP, $X / A$-like cells in the gastric fundus and duodenum remain in situ but are excluded from nutrient contact. Diet-induced weight loss increases ghrelin [19]. Yet, despite marked weight loss, postLRYGBP fasting acyl-ghrelin non-significantly decreased at $6 \mathrm{w}$ but rose towards baseline values by $12 \mathrm{w}$, whereas at $\mathrm{t} 30$ post-meal declined from pre-surgery at $6 \mathrm{w}$ and $12 \mathrm{w}$. Our results suggest that stomach fundus and duodenal ghrelinproducing cells contribute to circulating ghrelin postLRYGBP despite their exclusion from nutrient contact and that mechanisms independent from $X / A$-like cell nutrientsensing, for example the vagus nerve [39], may signal mealinduced ghrelin suppression. Chronaiou et al. provided direct evidence that fundus ghrelin-producing cells remain active post-LRYGBP by demonstrating superior decreases in ghrelin post-LRYGBP+ fundus resection versus LRYGBP with fundus preservation [23]. Interestingly Barazzoni et al. reported no change in total ghrelin, but significantly increased acylghrelin at 1, 3, 6 and 12 months post-LRYGBP [24]. Substantial differences in sample handling by addition of esterase inhibitor and plasma acidification in our study may underlie these discrepant findings.

LSG removes gastric fundus ghrelin-producing cells and accelerates nutrient delivery to duodenal ghrelin-producing cells by increasing gastric emptying [25-27]. After LSG, 40-50\% decreases in fasting total-ghrelin have been reported, sustained for up to 5 years post-surgery, with reductions in post-meal circulating ghrelin levels [21, 28, 40]. We also observed reductions in fasting acyl-ghrelin and acylghrelinAUC ${ }_{0-180}$ post-LSG. Moreover, as anticipated in view of the stomach fundus excision, LSG induced superior acylghrelin reductions than LRYGBP. Interestingly, despite removing the majority of the gastric fundus, fasting acylghrelin and acyl-ghrelinAUC ${ }_{0-180}$ declined by only $\sim 20$ $30 \%$ post-LSG. A possible explanation is that although the majority of ghrelin-producing cells are in the stomach fundus, circulating acyl-ghrelin may primarily originate from the duodenum. Alternatively, plasma acyl-ghrelin is highly regulated, and compensatory up-regulation of duodenal ghrelinproduction may occur.

PYY and GLP-1 are released from distal gut L-cells. Postmeal their levels rapidly rise, implicating a yet unknown neural and/or humoral mechanism to this initial release. Subsequent PYY and GLP-1 release results from L-cell nutrient-contact. LRYGBP and LSG increase gastric emptying [25-27, 41] but have different effects on gut nutrientpassage. LRYGBP excludes nutrients from foregut contact and expedites nutrient delivery to distal gut L-cells, which is suggested to augment hindgut hormone release; the 'hindgut theory'. LSG accelerates gastric emptying, reduces acid production and rapidly transits nutrients into the duodenum and proximal intestine, enhancing foregut stimulation [2]. Few studies have examined the effects of LRYGBP on circulating PYY3-36, the anorectic PYY-isoform. This is the 


\section{A}

Pre-operative hunger VAS, LRYGBP vs. LSG

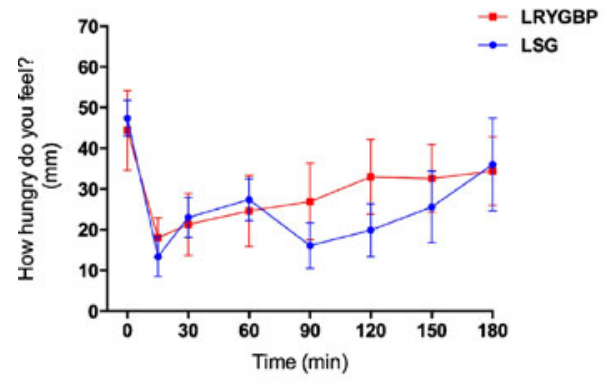

B

Pre-operative fullness VAS, LRYGBP vs. LSG

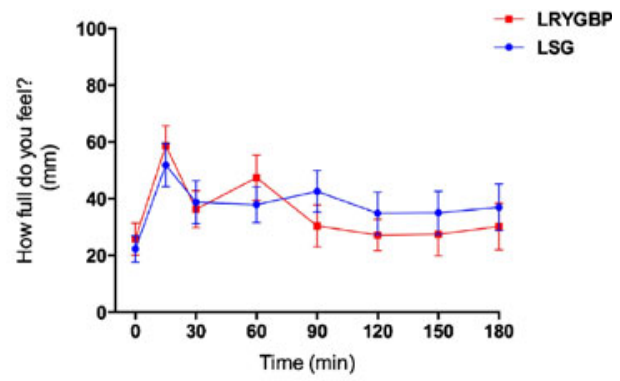

C

Pre-operative acyl-ghrelin, LRYGBP vs. LSG

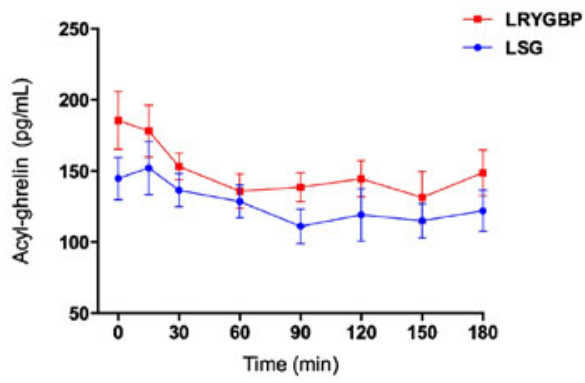

D

Pre-operative PYY3-36, LRYGBP vs. LSG

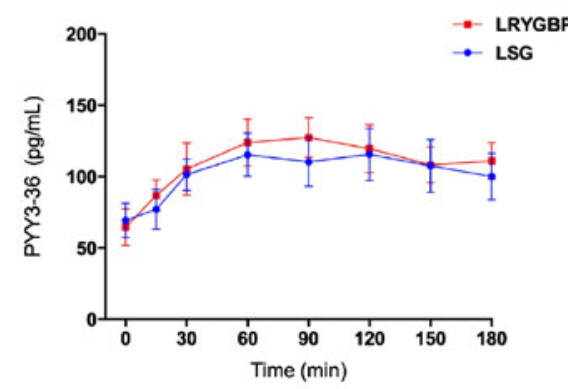

$\mathbf{F}$

Pre-operative glucose, LRYGBP vs. LSG

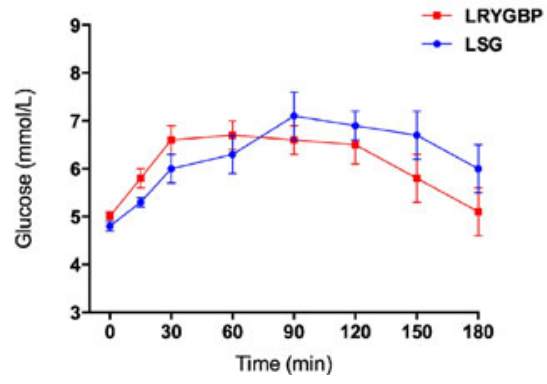

Fig. 1 Fasting and nutrient-stimulated appetite, gut hormones, glucose and insulin for the LRYGBP and LSG groups pre-operatively. (A) Temporal hunger visual analogue scales $(V A S)$ ratings for the LRYGBP (red, filled squares) and LSG (blue, filled circles). (B) Temporal fullness VAS for the LRYGBP (red, filled squares) and LSG (blue, filled circles) groups. (C) Plasma acyl-ghrelin temporal profile for the LRYGBP (red, filled squares) and LSG (blue, filled circles) groups. (D) Plasma PYY3-
E

Pre-operative active GLP-1, LRYGBP vs. LSG

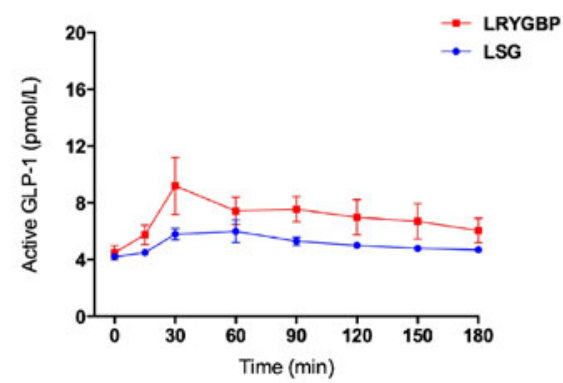

G

Pre-operative insulin, LRYGBP vs. LSG

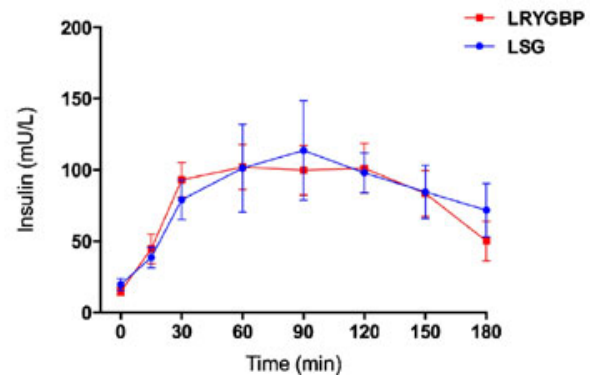

36 temporal profile for the LRYGBP (red, filled squares) and LSG (blue, filled circles) groups. (E) Plasma active GLP-1 temporal profile for the LRYGBP (red, filled squares) and LSG (blue, filled circles) groups. (F) Plasma glucose temporal profile for the LRYGBP (red, filled squares) and LSG (blue, filled circles) groups. (G) Plasma insulin temporal profile for the LRYGBP (red, filled squares) and LSG (blue, filled circles) groups. Results are expressed as mean $\pm \mathrm{SEM}$ 


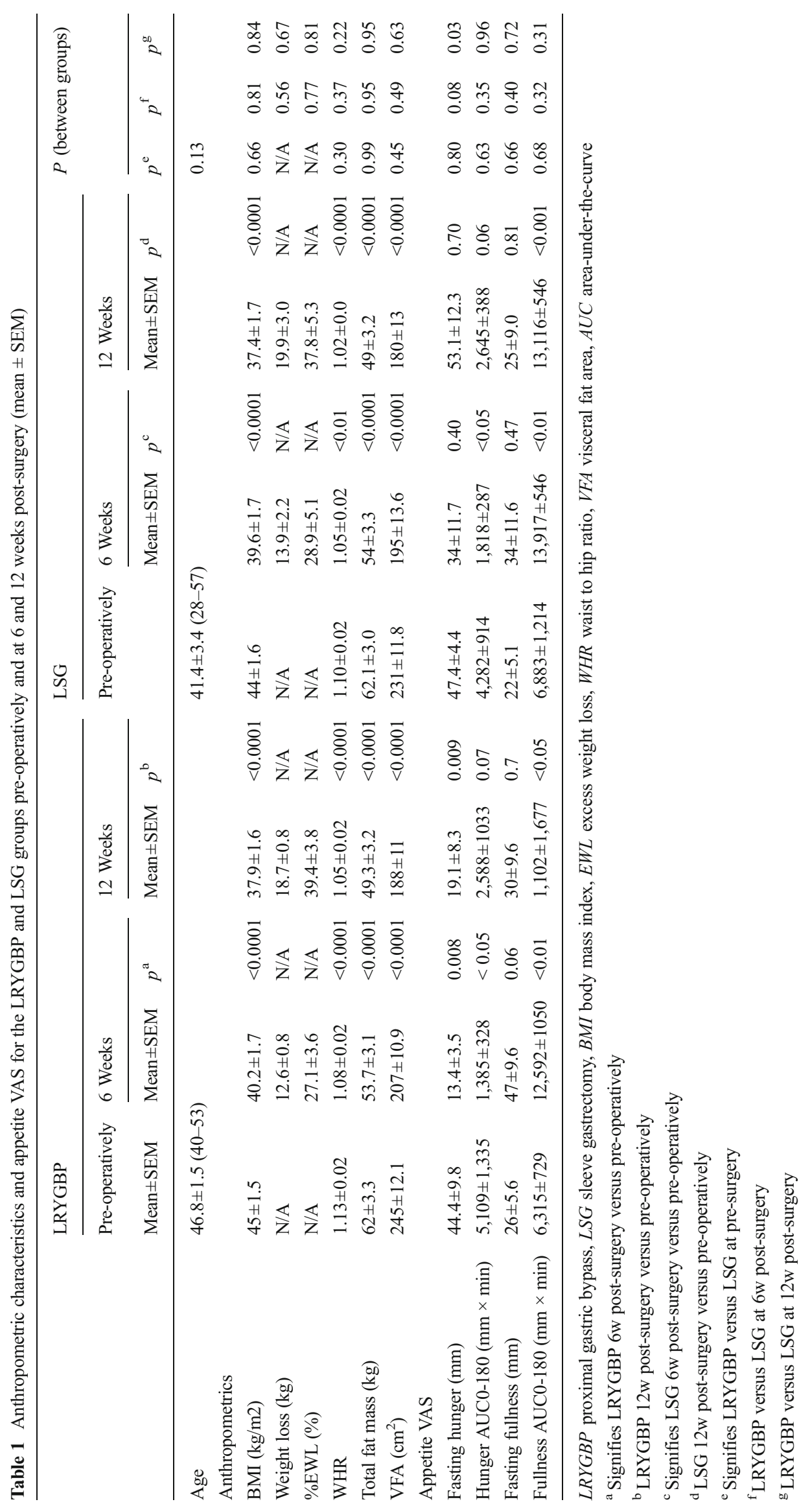




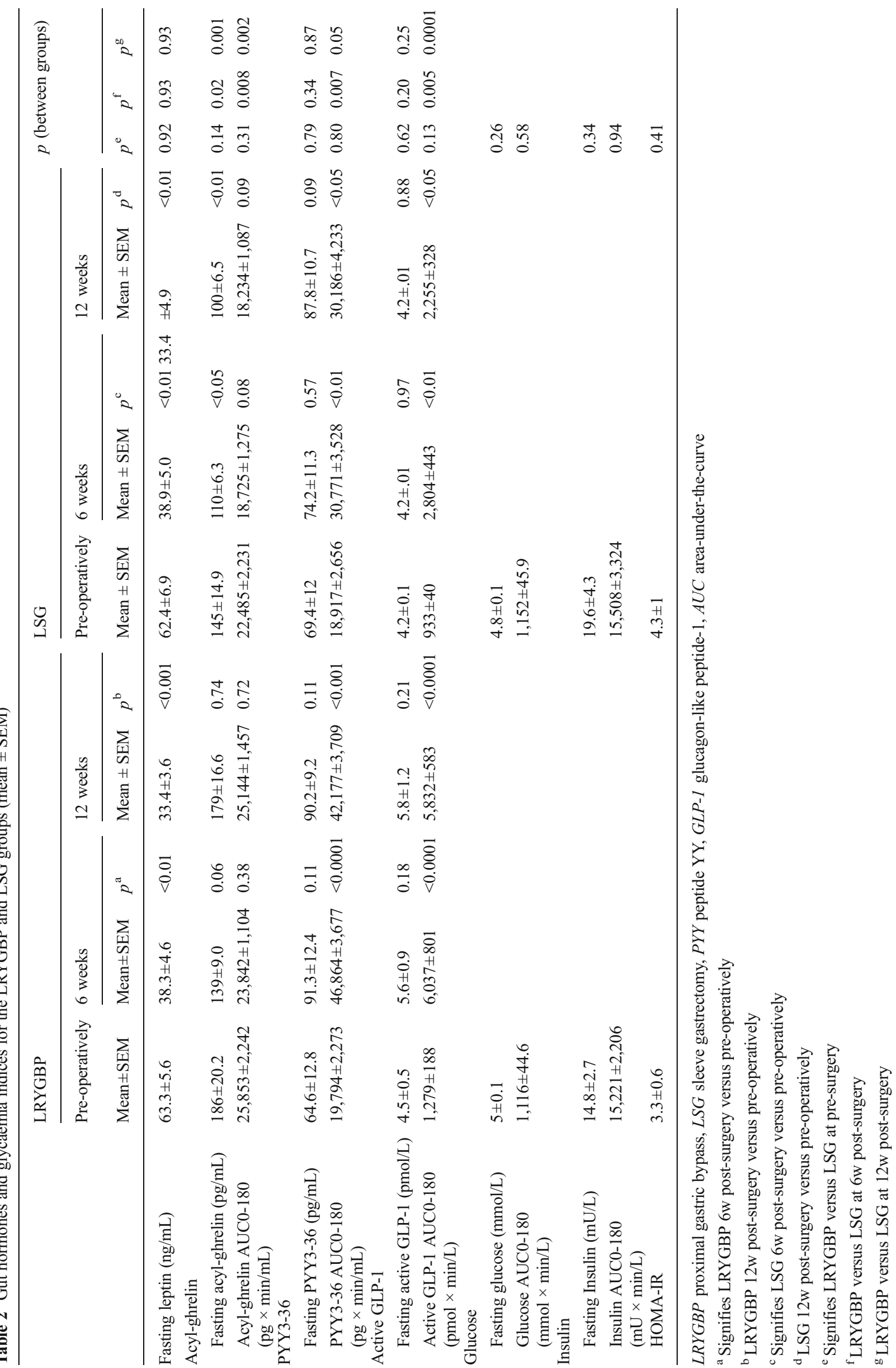


A

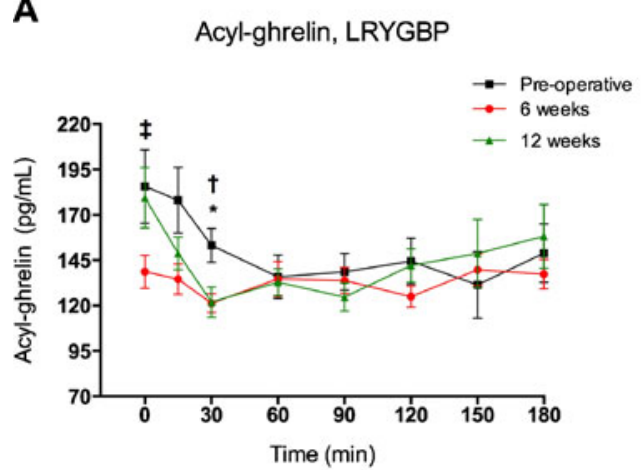

B

Acyl-ghrelin, LSG

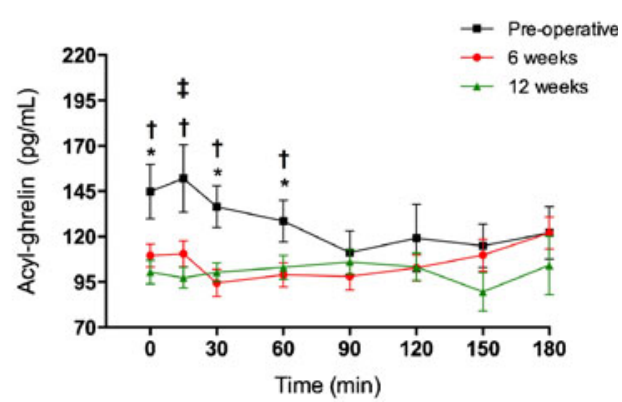

C

Acyl-ghrelin AUC 0-180 LRYGBP vs. LSG
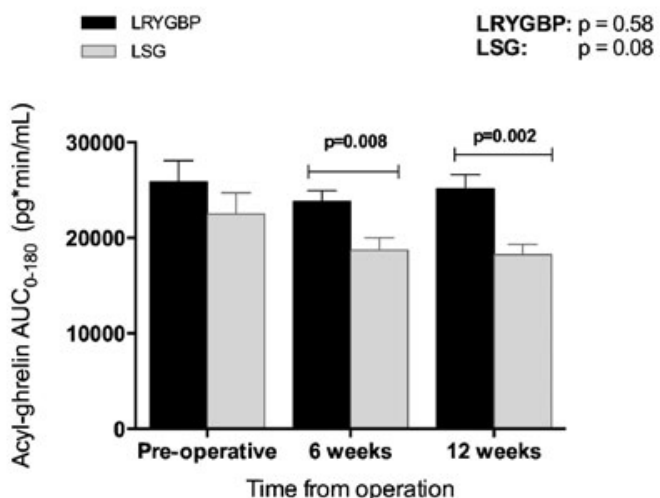

Fig. 2 The effects of LRYGBP and LSG on plasma fasting, mealstimulated acyl-ghrelin and acyl-ghrelinAUC ${ }_{0-180}$. Plasma acyl-ghrelin temporal profile in response to the test-meal for LRYGBP (a) and LSG (b) groups at pre-surgery (black, solid squares), and at $6 \mathrm{w}$ and $12 \mathrm{w}$ postoperatively (red, solid circles and green, solid triangles, respectively). c Acyl-ghrelinAUC ${ }_{0-180}$ for LRYGBP (black, solid columns) and LSG groups (grey, solid columns) at pre-surgery and at $6 \mathrm{w}$ and $12 \mathrm{w}$ post-

first report of plasma PYY3-36 post-LSG. LRYGBP and LSG markedly enhanced nutrient-stimulated PYY3-36, with, however, greater, more sustained post-prandial PYY3-36 release post-LRYGBP. These findings are in keeping with the procedural anatomical differences and suggest that factors originating from foregut and hindgut regulate PYY3-36 levels. Our findings of superior PYY336 enhancement post-LRYGBP versus LSG are in accord with those of Valderas et al. for total-PYY [32]. However, they are at odds with reports of comparable post-prandial total-PYY following LRYGBP and LSG [21, 29, 31]. Differences in PYY-isoforms assessed, sampling timepoints, subject standardization, sample handling and in subjects' HOMA-IR pre-surgery may account for these discrepancies.

Similarly to PYY3-36, both procedures augmented nutrient-stimulated active GLP-1 levels, with again greater, more sustained release observed post-LRYGBP. These operatively. Results are expressed as mean \pm SEM. ${ }^{*} p<0.05$, withingroup at $6 \mathrm{w}$ post-operatively compared to pre-surgery; ${ }^{\dagger} p<0.05$, within-group comparisons at $12 \mathrm{w}$ post-operatively versus pre-surgery; ${ }^{\star} p<$ 0.05 , within-group $6 \mathrm{w}$ versus $12 \mathrm{w}$ comparison. The $p$ values at the right upper corner of $\mathbf{c}$ indicate one-way ANOVA within-group analysis. Within-group Bonferroni post hoc and between-group $t$ test significance is indicated over the corresponding bars

findings are at odds with reports by Chambers et al. of similar increases in nutrient-stimulated active GLP-1 post-sleeve gastrectomy and gastric bypass in rats [8]. These discrepancies may be accounted for by structural inter-species rodenthuman stomach differences, which potentially affect gastric emptying post-surgery and hence gut hormone responses. Moreover, Chambers and colleagues measured active GLP-1 5 months post-surgery in weight-stable rats, whilst our studies were undertaken during the acute weight-loss phase. Peterli et al. have reported greater meal-stimulated active GLP-1 responses at 1 week and non-significant increases 12 weeks post-LRYGBP versus LSG [21]. In another study, they showed non-significantly greater active GLP-1 peak and active GLP-1AUC following LRYGBP [29]. Again, methodological and subject-related differences may underlie these discrepancies.

Despite greater reductions in the 'hunger hormone' acylghrelin post-LSG versus post-LRYGBP, paradoxically the 
A

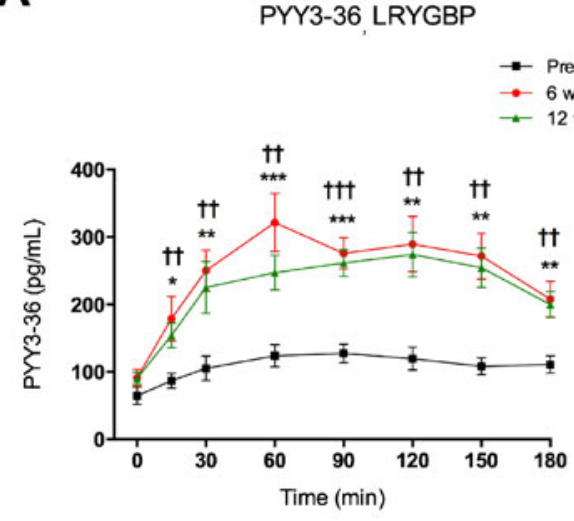

C

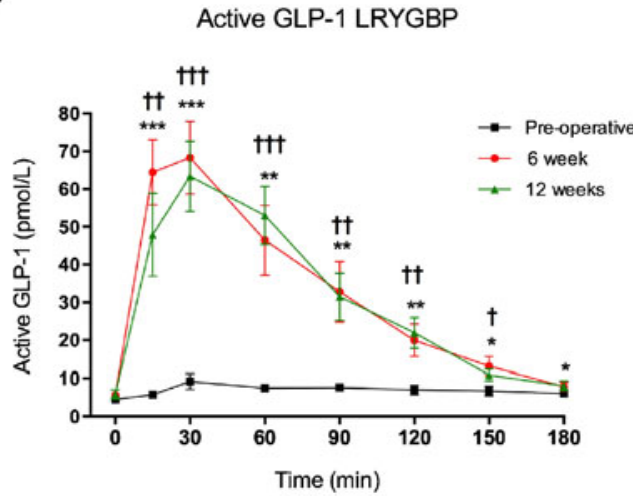

$\mathbf{E}$

PYY3-36 AUC $_{0-180}$ LRYGBP vs. LSG

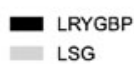

LRYGBP: $p<0.0001$
LSG: $\quad p=0.002$

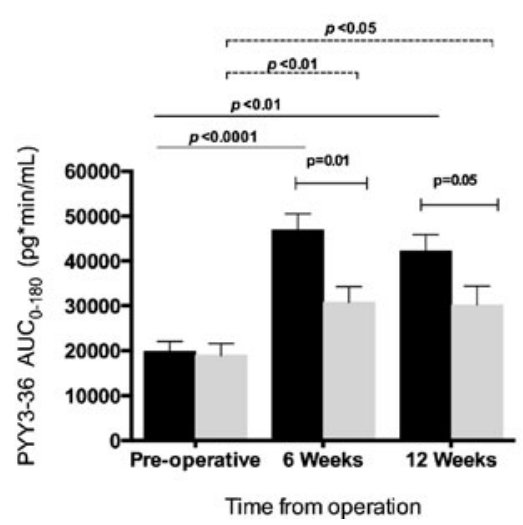

Fig. 3 The effects of LRYGBP and LSG on fasting, meal-stimulated plasma concentrations and area-under-the curve $\left(\mathrm{AUC}_{0-180}\right)$ for $\mathrm{PYY}_{3-36}$ and active GLP-1. Plasma PYY $3-36$ temporal profile in response to the test-meal for LRYGBP (a) and LSG (b) groups at pre-surgery (black, solid squares) and at $6 \mathrm{w}$ and $12 \mathrm{w}$ post-operatively (red, solid circles and green, solid triangles, respectively). Plasma active GLP-1 temporal profile in response to the test-meal for LRYGBP (c) and LSG (d) groups at pre-surgery (black, solid squares) and at $6 \mathrm{w}$ and $12 \mathrm{w}$ post-operatively (red, solid circles and green, solid triangles, respectively). $\mathrm{PYY}_{3-}$
B

PYY3-36, LSG

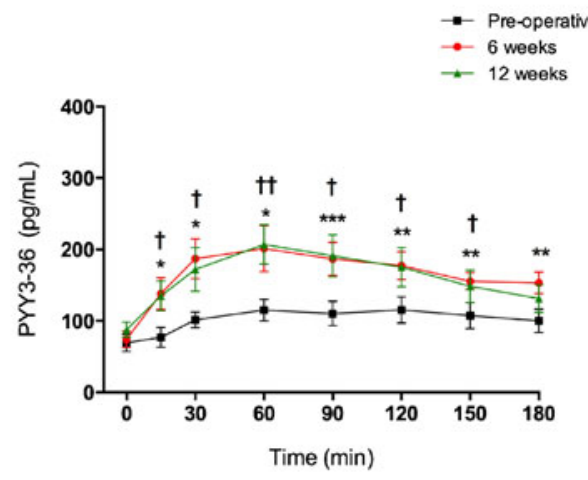

D

Active GLP-1, LSG

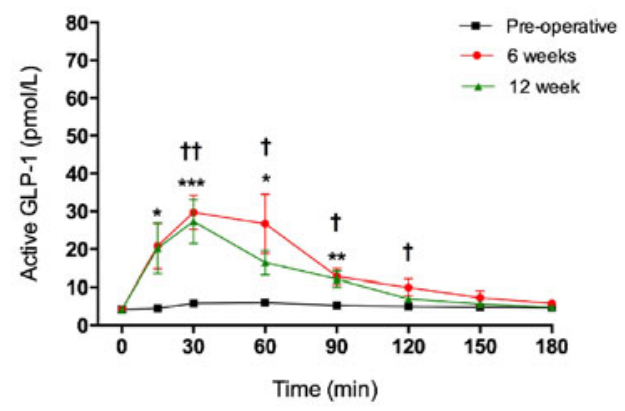

$\mathbf{F}$
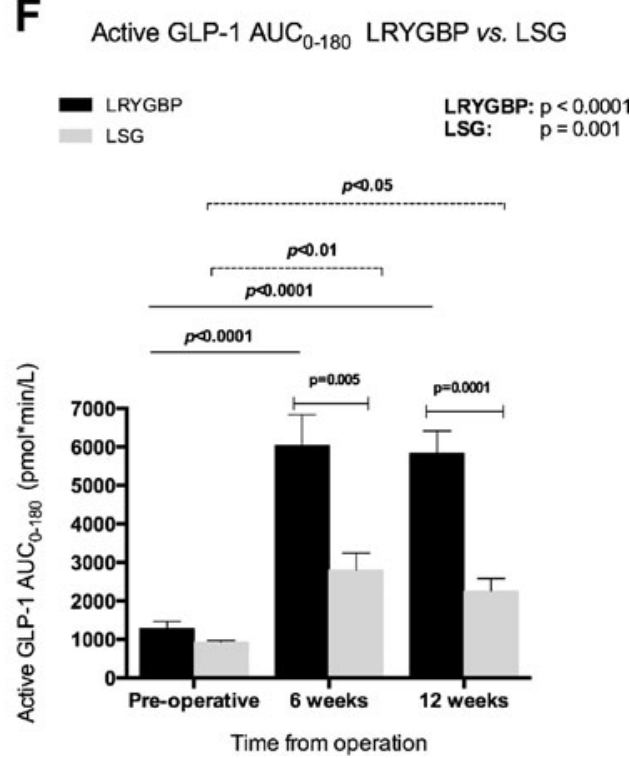

${ }_{36} \mathrm{AUC}_{0-180}(\mathbf{e})$ and active GLP-1 $\mathrm{AUC}_{0-180}(\mathbf{f})$ for LRYGBP (black, solid columns) and LSG groups (grey, solid columns) at pre-surgery and at $6 \mathrm{w}$ and $12 \mathrm{w}$ post-operatively. Results are expressed as mean \pm SEM. ${ }^{*} p<$ $0.05, * * p<0.01$ and $* * * p<0.001$ within-group at $6 \mathrm{w}$ post-operatively compared to pre-surgery. ${ }^{\dagger} p<0.05,{ }^{\dagger} p<0.01$ and ${ }^{\dagger \dagger \dagger} p<0.001$ for withingroup comparisons at $12 \mathrm{w}$ post-operatively versus pre-surgery. The $p$ values at the right upper corner of $\mathbf{e}$ and $\mathbf{f}$ indicate one-way ANOVA within-group analysis. Within-group Bonferroni post hoc and betweengroup $t$ test significance is indicated over the corresponding bars 
A

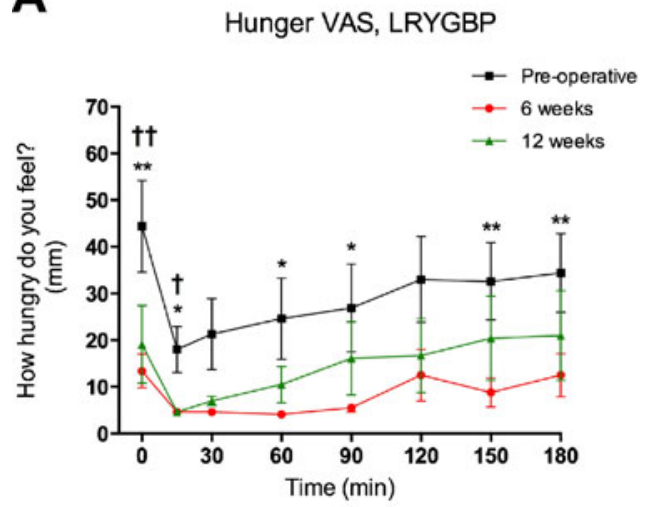

C

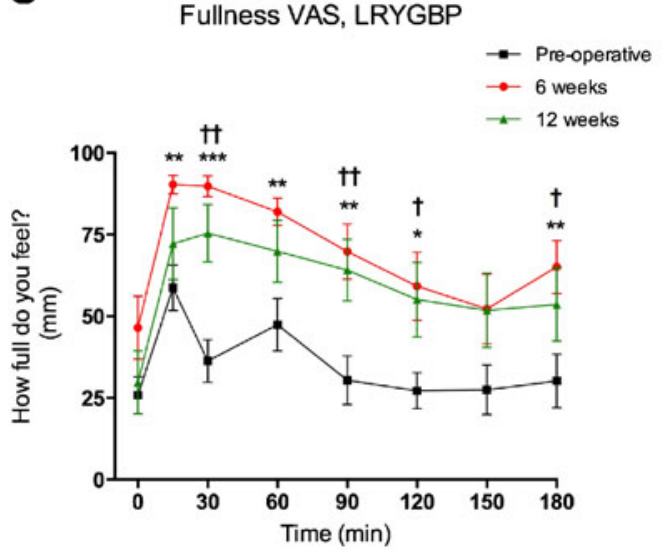

$\mathbf{E}$ Hunger AUC $_{0-180}$ LRYGBP vs. LSG

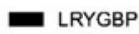
$\square$ LSG

\section{LRYGBP: $p=0.01$}

LSG: $\quad p=0.02$

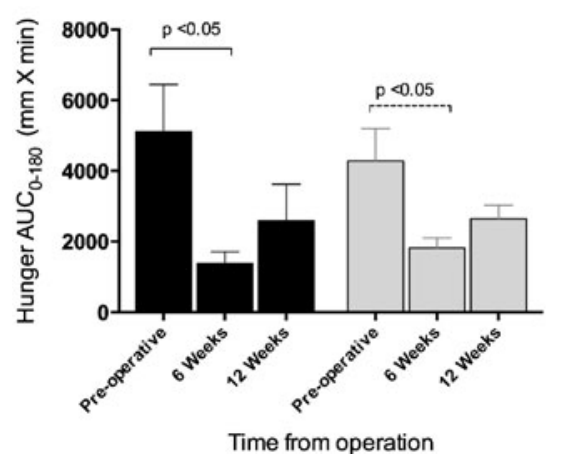

Fig. 4 The effects of LRYGBP and LSG on fasting, meal-stimulated and area-under the curve $\left(\mathrm{AUC}_{0-180}\right)$ for subjective hunger and fullness VAS ratings. Hunger VAS temporal profile in response to the test-meal for LRYGBP (a) and LSG (b) groups at pre-surgery (black, solid squares), and at $6 \mathrm{w}$ and $12 \mathrm{w}$ post-operatively (red, solid circles and green, solid triangles, respectively). Fullness VAS temporal profile in response to the test-meal for LRYGBP (c) and LSG (d) groups at pre-surgery (black, solid squares) and at $6 \mathrm{w}$ and $12 \mathrm{w}$ post-operatively (red, solid circles and green, solid triangles, respectively). HungerAUC $\mathrm{C}_{0-180}$ (e) and
B
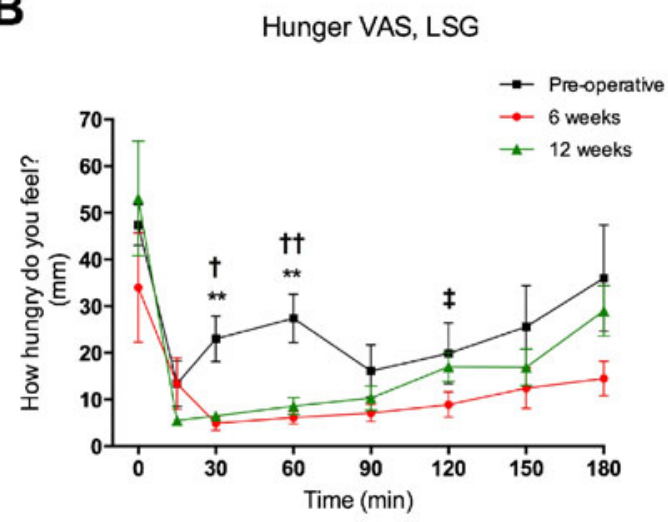

D
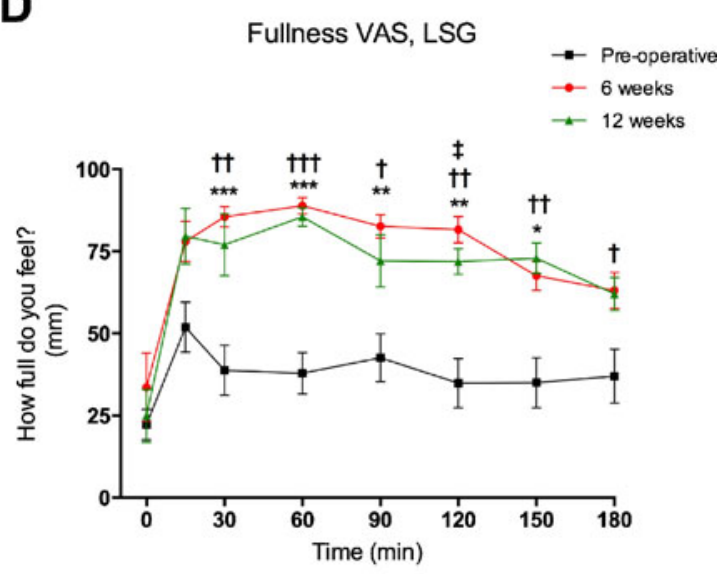

$\mathbf{F}$

Fullness $\mathrm{AUC}_{0-180}$ LRYGBP vs. LSG

$\begin{array}{ll}\text { LRYGBP } & \text { LRYGBP: } p=0.002 \\ \text { LSG } & \text { LSG: } \quad p=0.0001\end{array}$

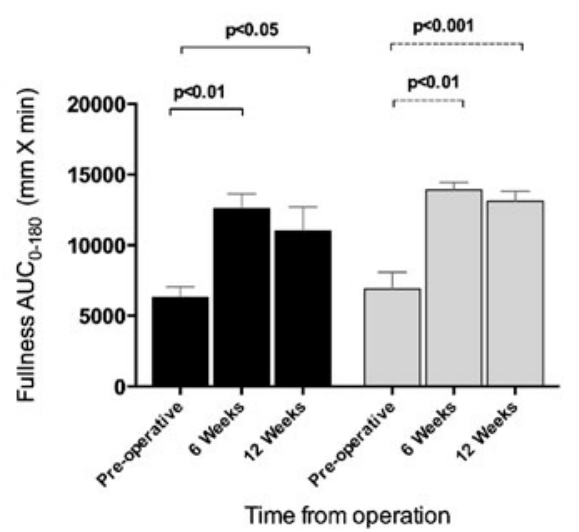

fullnessAUC $0-180$ (f) for LRYGBP (black, solid columns) and LSG groups (grey, solid columns) at pre-surgery and at $6 \mathrm{w}$ and $12 \mathrm{w}$ postoperatively. Results are expressed as mean \pm sem. ${ }^{*} p<0.05, * * p<0.01$ and $* * * p<0.001$ within-group at $6 \mathrm{w}$ post-operatively compared to presurgery. ${ }^{\dagger} p<0.05,{ }^{\dagger \dagger} p<0.01$ and ${ }^{\dagger \dagger} p<0.001$ for within-group comparisons at $12 \mathrm{w}$ post-operatively versus pre-surgery. The $p$ values at the right upper corner $\mathbf{e}$ and $\mathbf{f}$ indicate one-way ANOVA within-group analysis. Within-group Bonferroni post hoc and between-group $t$ test significance is indicated over the corresponding bars 
LRYGBP group exhibited lower fasting hunger; post-prandial hunger was reduced comparably by both procedures. We also observed similar post-operative increments in nutrientstimulated fullness perception in both groups. These findings again are slightly at odds with the accepted notion that PYY336 and active GLP-1 mediate satiety, as LRYGBP induced superior increases of these anorectic peptides and thus would be expected to result in greater satiety perception. These findings highlight that additional factors to active GLP-1 and PYY3-36 regulate satiety.

The novel finding of our study is the characterisation for the first time of the effects of LSG on circulating levels of the anorectic PYY-isoform, PYY3-36. Moreover, our study is the first to simultaneously measure bioactive forms of ghrelin, PYY and GLP-1 in the same patient cohort, while concurrently undertaking parallel appetite assessment. The main strength of our study is the use of validated subject-standardisation protocols, stringent sample processing [13], and tight groupmatching pre-operatively for the gut hormone confounders age [42], sex [43] and adiposity [44]. Furthermore, we studied patients without T2DM, dissecting out confounding effects of T2DM on the incretin effect [45], circulating PYY3-36 [5] and ghrelin [46]. The limitations of our study are our small sample sizes, non-randomization and limited follow-up. We studied females only as these represent the majority of patients undergoing bariatric surgical procedures in the UK, and future studies are needed to assess whether gender differences exist in postoperative gut hormone changes. Moreover, studies in patients with T2DM are required to examine whether their inferior weight-loss outcome post-bariatric surgery [47] results from altered/aberrant gut hormone responses. Future larger, randomised studies with longitudinal assessment of gut hormones, intestinal transit, glycaemic and anthropometric indices are required to further elucidate the mechanisms underlying the beneficial effects of LRYGBP and LSG in an attempt to develop novel, less invasive surgical and non-surgical T2DM and obesity treatments.

Acknowledgments We are indebted to our patients who participated in this study. We thank all the members of the UCLH Bariatric Group, past and present. This work was supported by the Rosetrees Trust, Medical Research Council, UCLH Charities and UCL/UCLH Comprehensive Biomedical Research Centre who received a proportion of funding from the Department of Health's NIHR Biomedical Research Centres funding scheme

Conflict of Interest All authors declare no conflict of interest.

Open Access This article is distributed under the terms of the Creative Commons Attribution License which permits any use, distribution, and reproduction in any medium, provided the original author(s) and the source are credited.

\section{References}

1. Sjostrom L, Peltonen M, Jacobson P, et al. Bariatric surgery and longterm cardiovascular events. JAMA. 2012;307:56-65.

2. Scott WR, Batterham RL. Roux-en-Y gastric bypass and laparoscopic sleeve gastrectomy: understanding weight loss and improvements in type 2 diabetes after bariatric surgery. Am J Physiol Regul Integr Comp Physiol. 2011;301:R15-27.

3. Morinigo R, Moize V, Musri M, et al. Glucagon-like peptide-1, peptide YY, hunger, and satiety after gastric bypass surgery in morbidly obese subjects. J Clin Endocrinol Metab. 2006;91:1735-40.

4. Laferrere B. Effect of gastric bypass surgery on the incretins. Diabetes Metab. 2009;35:513-7.

5. Olivan B, Teixeira J, Bose M, et al. Effect of weight loss by diet or gastric bypass surgery on peptide YY3-36 levels. Ann Surg. 2009;249:948-53.

6. Gould JC, Kent KC, Wan Y, et al. Perioperative safety and volume: outcomes relationships in bariatric surgery: a study of 32,000 patients. J Am Coll Surg. 2011;213:771-7.

7. Deitel M, Gagner M, Erickson AL, et al. Third international summit: current status of sleeve gastrectomy. Surg Obes Relat Dis. 2011;7: 749-59.

8. Chambers AP, Jessen L, Ryan KK, et al. Weight-independent changes in blood glucose homeostasis after gastric bypass or vertical sleeve gastrectomy in rats. Gastroenterology. 2011;141:950-8.

9. Paluszkiewicz R, Kalinowski P, Wroblewski T, et al. Prospective randomized clinical trial of laparoscopic sleeve gastrectomy versus open Roux-en-Y gastric bypass for the management of patients with morbid obesity. Wideochir Inne Tech Malo Inwazyjne. 2012;7:225-32.

10. Vidal P, Ramon JM, Goday A, et al. Laparoscopic gastric bypass versus laparoscopic sleeve gastrectomy as a definitive surgical procedure for morbid obesity. Mid-term results, Obes Surg. 2013;23: $292-9$.

11. Hillman JB, Tong J, Tschop M. Ghrelin biology and its role in weight-related disorders. Discov Med. 2011;11:521-8.

12. Hosoda H, Doi K, Nagaya N, et al. Optimum collection and storage conditions for ghrelin measurements: octanoyl modification of ghrelin is rapidly hydrolyzed to desacyl ghrelin in blood samples. Clin Chem. 2004;50:1077-80.

13. Chandarana K, Drew ME, Emmanuel J, et al. Subject standardization, acclimatization, and sample processing affect gut hormone levels and appetite in humans. Gastroenterology. 2009;136:2115-26.

14. Batterham RL, Heffron H, Kapoor S, et al. Critical role for peptide $\mathrm{YY}$ in protein-mediated satiation and body-weight regulation. Cell Metab. 2006;4:223-33.

15. Batterham RL, Bloom SR. The gut hormone peptide YY regulates appetite. Ann N Y Acad Sci. 2003;994:162-8.

16. Nadkarni PP, Costanzo RM, Sakagami M. Pulmonary delivery of peptide YY for food intake suppression and reduced body weight gain in rats. Diabetes Obes Metab. 2011;13:408-17.

17. Chelikani PK, Haver AC, Reidelberger RD. Intravenous infusion of peptide YY(3-36) potently inhibits food intake in rats. Endocrinology. 2005;146:879-88.

18. Sloth B, Holst JJ, Flint A, et al. Effects of PYY1-36 and PYY3-36 on appetite, energy intake, energy expenditure, glucose and fat metabolism in obese and lean subjects. Am J Physiol Endocrinol Metab. 2007;292:E1062-8.

19. Sumithran P, Prendergast LA, Delbridge E, et al. Long-term persistence of hormonal adaptations to weight loss. N Engl J Med. 2011;365:1597-604.

20. Valderas JP, Irribarra V, Rubio L, et al. Effects of sleeve gastrectomy and medical treatment for obesity on glucagon-like peptide 1 levels and glucose homeostasis in non-diabetic subjects. Obes Surg. 2011;21:902-9.

21. Peterli R, Wolnerhanssen B, Peters T, et al. Improvement in glucose metabolism after bariatric surgery: comparison of laparoscopic Roux- 
en-Y gastric bypass and laparoscopic sleeve gastrectomy: a prospective randomized trial. Ann Surg. 2009;250:234 41.

22. Morinigo R, Vidal J, Lacy AM, et al. Circulating peptide YY, weight loss, and glucose homeostasis after gastric bypass surgery in morbidly obese subjects. Ann Surg. 2008;247:270-5.

23. Chronaiou A, Tsoli M, Kehagias I, et al. Lower ghrelin levels and exaggerated postprandial peptide-YY, glucagon-like peptide-1, and insulin responses, after gastric fundus resection, in patients undergoing Roux-en-Y gastric bypass: a randomized clinical trial. Obes Surg. 2012;22:1761-70.

24. R. Barazzoni, A. Semolic, M.R. Cattin, M. Zanetti, G. Guarnieri. Acylated ghrelin limits fat accumulation and improves redox state and inflammation markers in the liver of high-fat-fed rats. Obesity (Silver Spring). 2013.

25. Melissas J, Daskalakis M, Koukouraki S, et al. Sleeve gastrectomy-a "food limiting" operation. Obes Surg. 2008;18:1251-6.

26. Baumann T, Kuesters S, Grueneberger J, et al. Time-resolved MRI after ingestion of liquids reveals motility changes after laparoscopic sleeve gastrectomy-preliminary results. Obes Surg. 2011;21:95-101.

27. J. Melissas, A. Leventi, I. Klinaki, et al. Alterations of global gastrointestinal motility after sleeve gastrectomy: a prospective study. Annals of Surgery. 2012.

28. Karamanakos SN, Vagenas K, Kalfarentzos F, et al. Weight loss, appetite suppression, and changes in fasting and postprandial ghrelin and peptide-YY levels after Roux-en-Y gastric bypass and sleeve gastrectomy: a prospective, double blind study. Ann Surg. 2008;247:401-7.

29. Peterli R, Steinert RE, Woelnerhanssen B, et al. Metabolic and Hormonal Changes After Laparoscopic Roux-en-Y Gastric Bypass and Sleeve Gastrectomy: a Randomized. Obes Surg: Prospective Trial; 2012.

30. Ramon JM, Salvans S, Crous X, et al. Effect of Roux-en-Y gastric bypass vs sleeve gastrectomy on glucose and gut hormones: a prospective randomised trial. J Gastrointest Surg. 2012;16:1116-22.

31. F. Romero, J. Nicolau, L. Flores, R. Casamitjana, A. Ibarzabal, A. Lacy, J. Vidal, Comparable early changes in gastrointestinal hormones after sleeve gastrectomy and Roux-En-Y gastric bypass surgery for morbidly obese type 2 diabetic subjects, Surg Endosc, (2012)

32. Valderas JP, Irribarra V, Boza C, et al. Medical and surgical treatments for obesity have opposite effects on peptide YY and appetite: a prospective study controlled for weight loss. J Clin Endocrinol Metab. 2010;95:1069-75.

33. Alam ML, Van der Schueren BJ, Ahren B, et al. Gastric bypass surgery, but not caloric restriction, decreases dipeptidyl peptidase-4 activity in obese patients with type 2 diabetes. Diabetes Obes Metab. 2011;13:378-81.
34. Kirchner H, Gutierrez JA, Solenberg PJ, et al. GOAT links dietary lipids with the endocrine control of energy balance. Nat Med. 2009; 15:741-5.

35. Gonzalez CR, Vazquez MJ, Lopez M, et al. Influence of chronic undernutrition and leptin on GOAT mRNA levels in rat stomach mucosa. J Mol Endocrinol. 2008;41:415-21.

36. Goebel-Stengel M, Hofmann T, Elbelt U, et al. The ghrelin activating enzyme ghrelin-O-acyltransferase (GOAT) is present in human plasma and expressed dependent on body mass index. Peptides. 2013;43: 13-9.

37. Gagner M, Deitel M, Kalberer TL, et al. The second international consensus summit for sleeve gastrectomy. Surg Obes Relat Dis. 2009;5:476-85.

38. Matthews DR, Hosker JP, Rudenski AS, et al. Homeostasis model assessment: insulin resistance and beta-cell function from fasting plasma glucose and insulin concentrations in man. Diabetologia. 1985;28:412-9.

39. Sundbom M, Holdstock C, Engstrom BE, et al. Early changes in ghrelin following Roux-en-Y gastric bypass: influence of vagal nerve functionality? Obes Surg. 2007;17:304-10.

40. Bohdjalian A, Langer FB, Shakeri-Leidenmuhler S, et al. Sleeve gastrectomy as sole and definitive bariatric procedure: 5 -year results for weight loss and ghrelin. Obes Surg. 2010;20:535-40.

41. Falken Y, Hellstrom PM, Holst JJ, et al. Changes in glucose homeostasis after Roux-en-Y gastric bypass surgery for obesity at day three, two months, and one year after surgery: role of gut peptides. J Clin Endocrinol Metab. 2011;96:2227-35.

42. Rigamonti AE, Agosti F, Compri E, et al. Anorexigenic postprandial responses of PYY and GLP1 to slow ice cream consumption: preservation in obese adolescents, but not in obese adults. Eur J Endocrinol. 2013;168:429-36.

43. Kim BJ, Carlson OD, Jang HJ, et al. Peptide YY is secreted after oral glucose administration in a gender-specific manner. J Clin Endocrinol Metab. 2005;90:6665-71.

44. Neary MT, Batterham RL. Gut hormones: implications for the treatment of obesity. Pharmacol Ther. 2009;124:44-56.

45. Nauck M, Stockmann F, Ebert R, et al. Reduced incretin effect in type 2 (non-insulin-dependent) diabetes. Diabetologia. 1986;29:46-52.

46. Katsuki A, Urakawa H, Gabazza EC, et al. Circulating levels of active ghrelin is associated with abdominal adiposity, hyperinsulinemia and insulin resistance in patients with type 2 diabetes mellitus. Eur J Endocrinol. 2004;151:573-7.

47. Campos GM, Rabl C, Mulligan K, et al. Factors associated with weight loss after gastric bypass. Arch Surg. 2008;143:877-83. discussion 884. 